\title{
OPEN Homology between SARS CoV-2 and human proteins
}

\author{
Vladimir Khavinson $\left(^{1,3}\right.$, Alexander Terekhov ${ }^{1}{ }^{1}$, Dmitry Kormilets $\mathbb{1}^{2}$ \& \\ Alexander Maryanovich ${ }^{1}{ }^{凶}$
}

An extremely high contagiousness of SARS CoV-2 indicates that the virus developed the ability to deceive the innate immune system. The virus could have included in its outer protein domains some motifs that are structurally similar to those that the potential victim's immune system has learned to ignore. The similarity of the primary structures of the viral and human proteins can provoke an autoimmune process. Using an open-access protein database Uniprot, we have compared the SARS CoV-2 proteome with those of other organisms. In the SARS CoV-2 spike (S) protein molecule, we have localized more than two dozen hepta- and octamers homologous to human proteins. They are scattered along the entire length of the $S$ protein molecule, while some of them fuse into sequences of considerable length. Except for one, all these $n$-mers project from the virus particle and therefore can be involved in providing mimicry and misleading the immune system. All hepta- and octamers of the envelope (E) protein, homologous to human proteins, are located in the viral transmembrane domain and form a 28-mer protein $\mathrm{E}_{14-41}$ VNSVLLF LAFVVFLLVTLAILTALRLCA. The involvement of the protein $\mathrm{E}$ in provoking an autoimmune response (after the destruction of the virus particle) seems to be highly likely. Some SARS CoV-2 nonstructural proteins may also be involved in this process, namely ORF3a, ORF7a, ORF7b, ORF8, and ORF9b. It is possible that ORF7b is involved in the dysfunction of olfactory receptors, and the $S$ protein in the dysfunction of taste perception.

The interaction of SARS CoV-2 with the host immune system is largely determined by the structural similarities between viral and host proteins. The studies of SARS CoV-2 are still focused on the S protein ${ }^{1}$.

An extremely high contagiousness of the coronavirus SARS CoV-2 indicates that during its evolution the virus developed the ability to deceive the innate immune system. The simplest way to achieve this ability would be to incorporate into its membrane the proteins that share structural similarity with those which the immune system of the potential victim has learnt to ignore. Probably, the virus borrowed some n-mers from bats or other mammals. Any motif of any mammalian protein was suitable for borrowing, if only the immune system considered it to be of its own.

The knowledge of the homology between the SARS CoV-2 and human proteins would help understand the mechanisms of mimicry at the moment of infection. The SARS CoV-2 proteins may simulate human proteins, mislead the immune system, and slow down its response.

However, mimicry is not the only process that is determined by the protein homology between the virus and host organism. After the inevitable destruction of the virus particle, the proteins or their domains, which were inside the virus until then, come into contact with the immune system. With some structural similarity, a part of the immune response will be directed against the proteins of the host organism, i.e., an autoimmune response will arise.

This study aimed to identify the human proteins which share a significant structural homology with the SARS CoV-2 proteins. We hope this information will be useful to the developers of vaccines against coronavirus.

Joshua Lederberg ${ }^{2}$ believed that "microbes and their human hosts constitute a superorganism." According to this, we considered the concept of "human proteins" as a combination of human own proteome and the proteomes of gut microbiota. We have paid particular attention to the proteins that are involved in the three functions that are almost necessarily affected in this disease, namely digestion, olfaction and taste.

\footnotetext{
${ }^{1}$ Mechinkov North-Western State Medical University, 47 Piskaryovsky Prosp., 195067 St. Petersburg, Russia. ${ }^{2}$ Kirov Military Medical Academy, St. Petersburg, Russia. ${ }^{3}$ Saint Petersburg Institute of Bioregulation and Gerontology, St. Petersburg, Russia. ${ }^{\square}$ email: atm52atm52@gmail.com
} 
S protein, 1273 aa

\section{Methods}

Using an open-access protein database Uniprot and our original computer program Ouroboros ${ }^{3}$, we compared the SARS CoV-2 proteome ${ }^{4}$ with those of other organisms. We also searched for a separate database of 75,777 human proteins ${ }^{5}$. The algorithm we used compares primary sequences of SARS CoV-2 and human proteins, presented in the form of a one-letter code. We performed a comparison of proteins by a consecutive search for regions of one protein in the others, which is essentially a standard task of finding a substring in a string. This algorithm is implemented in standard methods of many programming languages, including Python, in which the main program was coded. The URL to the source code is provided above ${ }^{3}$.

When assessing the homology between the viral and human proteins, we took into account the presence of the common 7-/8-mers and especially their fusion into longer sequences. For example, 7-dimensional viruses, one of which is homologous to the human protein A, and the other to the protein B, can "overlap" at the ends, forming regions of 8 to 14 amino acid residues in length.

\section{Results and discussion \\ Structural proteins. Spike glycoprotein. S protein, 1273 aa.}

MFVFLVLLPLVSSOCVNLTTRTOLPPAYTNSFTRGVYYPDKVFRSSVLHSTODLFLPFFSNVTWFHAIHVSGTNGTKRFDNPVLPFNDGVYFASTEKSNI IRG WIFGTTLDSKTQSLLIVNNATNVVIKVCEFQFCNDPFLGVYYHKNNKSWMESEFRVYSSANNCTFEYVSQPFLMDLEGKQGNEKNLREFVFKNIDGYFKIYSK HTPINLVRDLPQGFSALEPLVDLPIGINITRFQTLLALHRSYLTPGDSSSGWTAGAAAYYVGYLQPRTFLLKYNENGTITDAVDCALDPLSETKCTLKSFTVE KGIYQTSNFRVQPTESIVRFPNITNLCPFGEVFNATRFASVYAWNRKRISNCVADYSVLYNSASFSTFKCYGVSPTKLNDLCFTNVYADSFVIRGDEVROIAP GQTGKIADYNYKLPDDFTGCVIAWNSNNLDSKVGGNYNYLYRLFRKSNLKPFERDISTEIYQAGSTPCNGVEGFNCYFPLQSYGFQPTNGVGYQPYRVVVLSF ELLHAPATVCGPKKSTNLVKNKCVNFNFNGLTGTGVLTESNKKFLPFQQFGRDIADTTDAVRDPQTLEILDITPCSFGGVSVITPGTNTSNQVAVLYQDVNCT EVPVAIHADQLTPTWRVYSTGSNVFQTRAGCLIGAEHVNNSYECDIPIGAGICASYQTQTNSPRRARSVASQSI IAYTMSLGAENSVAYSNNSIAIPTNFTIS VTTEILPVSMTKTSVDCTMYICGDSTECSNLLLQYGSFCTQLNRALTGIAVEQDKNTQEVFAQVKQIYKTPPIKDFGGFNFSQILPDPSKPSKRSFIEDLLFN KVTLADAGFIKQYGDCLGDIAARDLICAQKFNGLTVLPPLLTDEMIAQYTSALLAGTITSGWTFGAGAALQIPFAMQMAYRFNGIGVTQNVLYENQKLIANQF NSAIGKIQDSLSSTASALGKLQDVVNQNAQALNTLVKQLSSNFGAISSVLNDILSRLDKVEAEVQIDRLITGRLQSLQTYVTQQLIRAAEIRASANLAATKMS ECVLGQSKRVDFCGKGYHLMSFPQSAPHGVVFLHVTYVPAQEKNFTTAPAICHDGKAHFPREGVFVSNGTHWFVTQRNFYEPQI ITTDNTFVSGNCDVVIGIV NNTVYDPLQPELDSFKEELDKYFKNHTSPDVDLGDISGINASVVNIQKEIDRLNEVAKNLNESLIDLQELGKYEQYIKWPWY IWLGFIAGLIAIVMVTIMLCC MTSCCSCLKGCCSCGSCCKFDEDDSEPVLKGVKLHYT

Hereinafter, regions homologous to human proteins are highlighted in red. Transmembrane tail $\mathrm{TM}_{1214-1237}$ is underlined.

In the S protein molecule, we localized more than two dozen of 7-/8-mers homologous to human proteins (Table 1).

Fragments homologous to human proteins are scattered along the entire length of the $\mathrm{S}$ protein molecule, and some of them fuse in sequences of considerable length, namely 10-mers SPRRARSVAS $680-689$, 11-mers GLTVLPPLLTD $_{857-867}$ and two closely spaced 7-mers NASVVNI ${ }_{1173-1179}$ and EIDRLNE $1182-1188$. Octamer RRARSVAS $682-689$ is located at the junction of the S1 and S2 subunits. All these n-mers stand out from the virus particles and may be involved in the effect of mimicry.

SARS CoV-2 can cause smell and taste dysfunction, as well as muscle injury ${ }^{6}$.

The 8-mer DEDDSEPV $1257-1264$, located in the cytoplasmic tail, can be released during the destruction of the virus particle and get involved in orchestrating the immune system's response, directing a part of it to the homologous 8-mer in human unconventional myosin- $\mathrm{XVI}_{1404-1421}$. The role of this mechanism in muscle dysfunction in coronavirus infection deserves a special investigation.

The 8-mer RRARSVAS ${ }_{682-689}$ is homologous to the amiloride-sensitive sodium channel subunit alpha ${ }_{201-208}$, which is involved in salt taste perception ${ }^{7}$.

With a high degree of probability, it can be argued that the $S$ protein is involved in the process of mimicry. It may also take some part in provoking an autoimmune response.

We have checked the S protein homology across10 species, specifically primates, bats and some other mammals. The results are presented in Table entitled Similarity of SARS CoV-2 spike glycoprotein structure with some mammalian proteins in the electronic attachement. Probably, attention should be paid to the homologous regions common to SARS CoV-2, humans, and bats. The data presented so far do not allow us to derive a more general rule.

MYSFVSEETGTLIVNSVLLFLAFVVFLLVTLAILTALRLCAYCCNIVNVSLVKPSFYVYSRVKNLNSSRVPDLLV

Envelope small membrane protein. E protein, 75 aa (transmembrane domain ${ }_{8-38}$ is underlined).

In the E protein molecule, we localized seven 7-mers and one 8-mer homologous to human proteins (Table 2).

A fragment of the $\mathrm{E}_{8-38}$ protein transmembrane domain can be represented as follows:

ETGTLIVNSVILELAFVVFLLVTLAILTALRLCA

The size of the letters (point size) corresponds to the frequency of the viral 7-/8-mers in the human proteome.

The protein $\mathrm{E}$ transmembrane domain contains 7-/8-mers, homologous to the proteins of some gut bacteria and even cereals, for example, corn, sorghum, wheat, and barley (Table 3). 


\begin{tabular}{|c|c|c|c|}
\hline Subunit & SARS CoV-2 $\mathrm{S}$ protein domain & In $\mathrm{S}$ protein & In human proteins \\
\hline \multirow{16}{*}{ S1 } & Signal peptide (N-terminus) $)_{1-13}$ & None & - \\
\hline & \multirow{8}{*}{$\mathrm{N}$-terminus domain $\mathrm{NTD}_{14-305}$} & DKVFRSS $_{40-46}$ & Zinc finger protein $528_{275-281}$ \\
\hline & & FLPFFSN $_{55-61}$ & OTU domain-containing protein $6 \mathrm{~A}_{185-191}$ \\
\hline & & VSGTNGT $_{70-76}$ & Lysosome-associated membrane glycoprotein $1_{171-177}$ \\
\hline & & SLLIVNN $_{16-122}$ & ATP-binding cassette sub-family A member $10_{825-831}$ \\
\hline & & FKNLREF $_{186-192}$ & Isovaleryl-CoA dehydrogenase, mitochondrial $77-83$ \\
\hline & & TRFQTLI $236-242$ & Disheveled-associated activator of morphogenesis $2_{251-257}$ \\
\hline & & KIYSKHT $_{202-208}$ & Uncharacterized protein Clorf105 \\
\hline & & SSSGWTA $_{254-260}$ & Uncharacterized protein KIAA1109 (Fragment) ${ }_{610-616}$ \\
\hline & Uncharacterized fragment $t_{306-318}$ & None & - \\
\hline & \multirow{2}{*}{ Receptor-binding domain $\mathrm{RBD}_{319-541}$} & $\mathrm{KLNDLCF}_{386-392}$ & Interleukin- ${ }_{149-155}$ \\
\hline & & DEVRQIA $_{405-411}$ & Histone-lysine $\mathrm{N}$-methyltransferase $2 \mathrm{C}_{4530-4536}$ \\
\hline & \multirow{4}{*}{ Uncharacterized fragment $t_{542-787}$} & VYSTGSN $_{635-641}$ & Neural cell adhesion molecule L1-like protein ${ }_{341-347}$ \\
\hline & & IGAGICA $_{666-672}$ & Hepatitis A virus cellular receptor $2_{205-211}$ \\
\hline & & SPRRARS $_{680-686}$ & Hermansky-Pudlak syndrome 1 protein $_{258-264}$ \\
\hline & & RRARSVAS $_{682-689}$ & Amiloride-sensitive sodium channel subunit alpha $201-208$ \\
\hline \multirow{14}{*}{ S2 } & Fusion peptide $\mathrm{FP}_{788-806}$ & None & - \\
\hline & \multirow{3}{*}{ Uncharacterized fragment $807-911$} & VTLADAG $_{826-832}$ & Non-receptor tyrosine-protein kinase $\mathrm{TNK} 1_{440-446}$ \\
\hline & & GLTVLPP $_{857-863}$ & FH1/FH2 domain-containing protein $3_{972-978}$ \\
\hline & & LPPLLTD $_{861-867}$ & $\begin{array}{l}\text { Maestro heat-like repeat-containing protein family mem- } \\
\text { ber } 9_{250-256}\end{array}$ \\
\hline & \multirow{2}{*}{ Heptapeptide repeat sequence $1 \mathrm{HR} 1_{912-984}$} & SSTASAL $_{939-945}$ & $40 \mathrm{~S}$ ribosomal protein $\mathrm{S} 13_{143-149}$ \\
\hline & & LVKQLSS $_{962-968}$ & E3 SUMO-protein ligase PIASl $_{284-290}$ \\
\hline & \multirow{4}{*}{ Uncharacterized fragment ${ }_{985-1162}$} & KVEAEVQ $Q_{986-974}$ & Emilin-3 $3_{625-631}$ \\
\hline & & TGRLQSL $_{998-1004}$ & Neuron navigator $3_{1610-1616}$ \\
\hline & & LIRAAE I $_{1012-1018}$ & Unconventional myosin-XVIIIa ${ }_{1352-1358}$ \\
\hline & & LDKYFKN $_{1152-1158}$ & Follistatin-related protein $1_{149-155}$ \\
\hline & \multirow{2}{*}{ Heptapeptide repeat sequence $2 \mathrm{HR} 2_{1163-1213}$} & NASVVNI $I_{1173-1179}$ & Thyroid adenoma-associated protein ${ }_{1022-1028}$ \\
\hline & & EIDRLNE $_{1182-1188}$ & Protein SETSIP ${ }_{64-70} ;$ Protein SET $_{54-60}$ \\
\hline & Transmembrane tail $\mathrm{TM}_{1214-1237}$ & None & - \\
\hline & Cytoplasm tail $\mathrm{CT}_{1238-1273}$ & DEDDSEPV $_{1257-1264}$ & Unconventional myosin- $\mathrm{XVI}_{1404-1421}$ \\
\hline
\end{tabular}

Table 1. Localization of homologous 7-/8-mers in the $S$ protein and human proteins.

\begin{tabular}{|c|c|c|}
\hline E protein domains $\mathrm{s}^{\mathrm{a}}$ & In E protein & In human proteins \\
\hline Signal peptide (N-terminus domain) $)_{1-7}$ & None & - \\
\hline \multirow{8}{*}{ Transmembrane domain $8-38$} & VNSVLLF $_{14-20}$ & Heterogeneous nuclear ribonucleoprotein $\mathrm{L}_{191-197}$ \\
\hline & VNSVLLFL $_{14-21}$ & Ran-binding protein $6_{409-416}$ \\
\hline & NSVLLFL $_{15-21}$ & Lysosomal amino acid transporter 1 homolog ${ }_{133-139}$ \\
\hline & SVLLFLA $_{16-22}$ & Cytochrome P450 2B6 $6_{4-10}$; Cytochrome P450 2B7 ${ }_{4-10}$; GPI ethanolamine phosphate transferase $3_{5-11}$ \\
\hline & LAFVVFL $_{21-27}$ & Solute carrier family 15 member $4_{235-241}$ \\
\hline & VFLLVTL $_{25-31}$ & Alpha-(1,3)-fucosyltransferase $10_{20-26}$ \\
\hline & LAILTAL $_{31-37}$ & $\begin{array}{l}\text { Transient receptor potential cation channel subfamily M member } 6_{394-400} \text {; Transient receptor potential cation channel } \\
\text { subfamily M member } 3_{465-471}\end{array}$ \\
\hline & TALRLCA $_{35-41}{ }^{\mathrm{b}}$ & Protein disulfide-isomerase $\mathrm{TMX}_{8-14}$ \\
\hline Internal domain $_{39-75}$ & None & - \\
\hline
\end{tabular}

Table 2. Localization of homologous 7-/8-mers in the E protein and human proteins. ${ }^{a}$ Domain boundaries see in ${ }^{8}$. ${ }^{b}$ Heptamer TALRLCA $A_{35-41}$ is located at the junction of the transmembrane domain ${ }_{8-38}$ and internal domain $_{39-75}$.

The simulation targets may have been the proteins synthesized by a macroorganism itself or by its normal gut microbiota.

All protein E 7-/8-mers, homologous to proteins of humans, gut bacteria and cereals, are located in the transmembrane domain of the virus and form the 28 -mer protein $\mathrm{E}_{14-41}$. A random selection of 28 amino acid residues in a row would require an astronomical number of iterations: $20^{28}=2.7 \cdot 10^{36}$. 


\begin{tabular}{|c|c|}
\hline In $\mathrm{E}$ protein & In bacterial and plant proteins \\
\hline AFVVFLLV $_{22-29}$ & 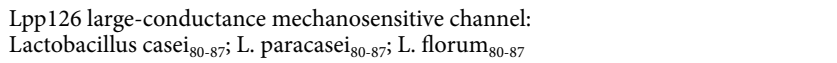 \\
\hline TLAILTA $_{30-36}$ & $\begin{array}{l}\text { Uncharacterized proteins: } \\
\text { Zea mays }_{90-164} ; \text { Sorghum bicolor }_{97-127} ; \text { Triticum aestivum }_{116-190} ; \text { Hordeum vulgare }_{87-161}\end{array}$ \\
\hline
\end{tabular}

Table 3. Localization of some of homologous 7-/8-mers in the E protein and human gut proteome.

\begin{tabular}{|c|c|}
\hline In $\mathrm{M}$ protein & In human proteins \\
\hline VEELKKL $_{10-16}$ & Glutaredoxin-related protein 5 , mitochondrial $_{135-141}$ \\
\hline EELKKLL $_{11-17}$ & GDP-fucose protein O-fucosyltransferase $2_{340-346}$ \\
\hline ELKKLLE $_{12-18}$ & Cullin- $1_{335-341}$ \\
\hline LKKLLEQ $Q_{13-19}$ & Filamin-A-interacting protein $1_{211-217}$ \\
\hline LLESELV $133-139$ & Leucine-rich repeat-containing protein $71_{439-445}$ \\
\hline AGDSGFA $_{188-194}$ & Myosin-14 $359-365$ \\
\hline
\end{tabular}

Table 4. Localization of homologous 7-mers in the M protein and human proteins.

The involvement of the E protein in mimicry is hardly possible, but its implication in provoking an autoimmune response (after the destruction of the virus particle) seems very likely.

As a major target, the viral $\mathrm{E}$ protein has usually been used for the development of vaccines, specifically against HIV $-1^{9}$, Dengue virus ${ }^{10}$, hepatitis B virus ${ }^{11}$, SARS CoV-2 ${ }^{12}$ and many other viruses. A deletion of the SARS-CoV E protein reduces pathogenicity and mortality in laboratory animals ${ }^{13}$. In the transmembrane domain of the SARS-CoV E protein, specific critical virulence-determining features have been identified ${ }^{14}$.

MADSNGTITVEELKKLLEQWNLVIGFLFLTWICLLQFAYANRNRFLYIIKLIFLWLLWPVTLACFVLAAVYRINWITGGIAIAMACL VGLMWLSYFIASFRLFARTRSMWSFNPETNILLNVPLHGTILTRPLLESELVIGAVILRGHLRIAGHHLGRCDIKDLPKEITVATSR TLSYYKLGASQRVAGDSGFAAYSRYRIGNYKLNTDHSSSSDNIALLVQ

Membrane protein. Membrane protein, 222 aa.

In the $\mathrm{M}$ protein molecule, we localized six 7-mers homologous to human proteins (Table 4).

A N-terminus fragment ${ }_{1-19}$ of the $\mathrm{M}$ protein can be represented as follows:

\section{MADSNGTITVEELKKLLEQWNLVIGFLF}

In the protein $\mathrm{M}$, four 7-dimensional homologues of human proteins are fused into 10-mer VEELKKLLEQ ${ }_{10-19}$, the hydrophilic composition of which indicates a possible contact with the external environment, i.e., with the host's immune system, and the involvement in mimicry.

Outside of the 10-mer, we found only two homologous 7-mers. It is unlikely that the M protein is involved in provoking an autoimmune response (after the destruction of the virus particle).

MSDNGPQNQRNAPRITFGGPSDSTGSNQNGERSGARSKQRRPQGLPNNTASWFTALTQHGKEDLKFPRGQGVPINTNSSPDDQ IGYYRRATRRIRGGDGKMKDLSPRWYFYYLGTGPEAGLPYGANKDGI IWVATEGALNTPKDHIGTRNPANNAAIVLQLPQGTT LPKGFYAEGSRGGSQASSRSSSRSRNSSRNSTPGSSRGTSPARMAGNGGDAALALLLLDRLNQLESKMSGKGQQQQGQTVTKK SAAEASKKPRQKRTATKAYNVTQAFGRRGPEQTQGNFGDQELIRQGTDYKHWPQIAQFAPSASAFFGMSRIGMEVTPSGTWLT YTGA IKLDDKDPNFKDQVILLNKH I DAYKTFPPTEPKKDKKKKADETQALPQRQKKQQTVTLLPAADLDDFSKQLQQSMSSAD STQA

Nucleoprotein. Nucleoprotein, 419 aa.

In the $\mathrm{N}$ protein molecule, we localized eleven 7 -mers homologous to human proteins (Table 5).

The $\mathrm{N}$ protein is located completely inside the virus particle and cannot be involved in mimicry. All heptamers homologous to human proteins form several rather long fragments, including the 13-mer SKQLQQSMSSADS $404-416$ and 10-mer AEGSRGGSQA $173-182$, which increases the likelihood of the protein involvement in provoking an autoimmune response.

Nonstructural proteins. All non-structural proteins of SARS CoV-2 are located completely inside the virus particle and, by definition, cannot be involved in the process of mimicry. It remains to consider the possibility of their implication in provoking an autoimmune process. 


\begin{tabular}{|c|c|}
\hline In $\mathrm{N}$ protein & In human proteins \\
\hline RPQGLPN $_{41-47}$ & GATOR complex protein WDR59 $9_{757-763}$ \\
\hline RGQGVPI $_{68-74}$ & Putative uncharacterized protein encoded by LINC00346 $6_{154-160}$ \\
\hline NSSPDDQ $Q_{77-83}$ & NEDD4-binding protein $2_{154-160}$ \\
\hline GKMKDLS $_{99-105}$ & Chromodomain-helicase-DNA-binding protein 1-like $_{770-776}$ \\
\hline VLQLPQG ${ }_{157-163}$ & Prestin $_{92-98}$ \\
\hline AEGSRGG $_{173-179}$ & snRNA-activating protein complex subunit $3_{2-8}$ \\
\hline SRGGSQA $_{176-182}$ & Ras-associating and dilute domain-containing protein ${ }_{886-892}$ \\
\hline $\mathrm{KADETQA}_{375-381}$ & Myopalladin $_{90-96}$ \\
\hline LLPAADL $_{394-400}$ & Probable E3 ubiquitin-protein ligase HERC1 $1_{1098-1104}$ \\
\hline SKQLQQS $404-410$ & Codanin-1 $1_{259-265}$ \\
\hline SMSSADS $_{410-416}$ & Protein PRRC2B $\mathrm{B}_{416-422}$ \\
\hline
\end{tabular}

Table 5. Localization of homologous 7 -mers in the $\mathrm{N}$ protein and human proteins.

\begin{tabular}{|l|l|}
\hline In ORF3a protein & In human proteins \\
\hline VGVALLA $_{48-54}$ & Manganese-transporting ATPase $13 \mathrm{Al}_{876-882}$ \\
\hline LLVAAGL $_{95-101}$ & Glycerophosphoinositol inositolphosphodiesterase GDPD2 ${ }_{129-135}$ \\
\hline KCRSKNP $_{132-138}$ & Vacuolar protein sorting-associated protein $13 \mathrm{~A}_{2066-2972}$ \\
\hline SVTSS IV $_{162-168}$ & Protein piccolo \\
T2779-2785 \\
\hline TQLSTDT $_{217-223}$ & Septin-14 \\
\hline
\end{tabular}

Table 6. Localization of homologous 7-mers in the ORF3a protein and human proteins.

\begin{tabular}{|l|l|}
\hline In ORF7a protein & In human proteins \\
\hline VAAIVF $I_{104-110}$ & Transmembrane protein $255 \mathrm{~B}_{86-92}$ \\
\hline FTLKRKT $_{114-120}$ & Cytosolic 5 '-nucleotidase $3 \mathrm{~A}_{36-42}$ \\
\hline
\end{tabular}

Table 7. Localization of homologous 7-mers in the ORF7a protein and human proteins.

MDLFMRIFTIGTVTLKQGEIKDATPSDFVRATATIPIQASLPFGWLIVGVALLAVFQSASKIITLKKRWQLALSKGVHFVCNLLLLF VTVYSHLLLVAAGLEAPFLYLYALVYFLQS INFVRI IMRLWLCWKCRSKNPLLYDANYFLCWHTNCYDYCIPYNSVTSSIVITSGDG TTSPISEHDYQIGGYTEKWESGVKDCVVLHSYFTSDYYQLYSTQLSTDTGVEHVTFFIYNKIVDEPEEHVQIHTIDGSSGVVNPVME PIYDEPTTTTSVPL

ORF3a protein. ORF3a protein, 275 aa.

In the ORF3a protein molecule, we localized five 7-mers homologous to human proteins (Table 6).

The 7-mers scattered along the entire length of its molecule do not form long n-mers anywhere else. ORF3a does not appear to be involved in provoking an autoimmune response.

MKI ILFLALITLATCELYHYQECVRGTTVLLKEPCSSGTYEGNSPFHPLADNKFALTCFSTQFAFACPDGVKHVYQLRARSVSPKLF IRQEEVQELYSPIFLIVAAIVFITLCFTLKRKTE

ORF7a protein. ORF7a 121 aa.

In the ORF7a protein molecule, we found two 7-mers homologous to human proteins and located in close proximity to each other (Table 7).

It is possible that ORF7a is involved in provoking an autoimmune response.

\section{MIELSLIDFYLCFLAFLLFLVLIMLIIFWFSLELQDHNETCHA}

ORF7b protein. ORF7b protein, 43 aa.

In this polypeptide, we found only one 7-mer homologous to the human protein (Table 8).

ORF7b may be involved in provoking an autoimmune response, contributing to olfactory dysfunction. 


\begin{tabular}{|l|l|}
\hline In ORF7b protein & In human protein \\
\hline IIFWFSL $_{26-32}$ & Olfactory receptor $7 \mathrm{D} 4_{151-157}$ \\
\hline
\end{tabular}

Table 8. Localization of the homologous 7-mer in ORF7b and a human protein.

\begin{tabular}{|l|l|}
\hline In ORF8 protein & In human proteins \\
\hline LVFLGII $_{4-10}$ & Zinc finger protein $486_{49-55}$ \\
\hline LGIITTV $_{7-13}$ & D-2-hydroxyglutarate dehydrogenase, mitochondrial \\
262-268 \\
\hline KLGSLVV $_{94-100}$ & Sodium leak channel non-selective protein \\
\hline
\end{tabular}

Table 9. Localization of homologous 7-mers in the ORF8 protein and human proteins.

\begin{tabular}{|c|c|}
\hline In ORF9b protein & In human proteins \\
\hline$L V D P Q I Q L_{14-21}$ & Valine-tRNA ligase, mitochondrial ${ }_{996-1002}$ \\
\hline MENAVGR $_{26-32}$ & Neprilysin $_{419-425}$ \\
\hline LGSPLSL $_{48-54}$ & Stress-responsive DNAJB4-interacting membrane protein $1_{37-43}$ \\
\hline GSPLSLN $_{49-55}$ & E3 ubiquitin-protein ligase HERC $2_{4533-4539}$ \\
\hline TEELPDE $_{84-90}$ & KH homology domain-containing protein $4_{465-471}$ \\
\hline ELPDEFVV $_{86-93}$ & Maestro heat-like repeat-containing protein family member $2 \mathrm{~B}_{103-110}$ \\
\hline
\end{tabular}

Table 10. Localization some of homologous 7-/8-mers in ORF9b protein and human proteins.

ORF8 protein. ORF8 protein, 121 aa.

The primary structure of SARS-CoV-2 ORF8 is close to that of bat RaTG13-CoV ${ }^{15}$. In this polypeptide, there are three 7-mers homologous to human proteins (Table 9).

Due to the fusion of two 7-mers into 10-mer LVFLGIITTV ${ }_{4-13}$, the ORF8 protein can be involved in provoking an autoimmune response.

ORF9b protein. ORF9b protein, 97 aa.

In the ORF9b protein molecule, we localized six 7-/8-mers, homologous to human proteins (Table 10).

Some of these 7-/8-mers merge into larger n-mers TEELPDEFVV $84-93$ and LGSPLSLN $48-55$.

Octamer ELPDEFVV $86-93$ is homologous to the Maestro heat-like repeat-containing protein family member 2B (Fig. 1), which may play a role in the sperm capacitation ${ }^{16}$. Male reproductive dysfunction was proposed as a likely consequence of COVID-19 17 .

After the destruction of the virus particle, ORF9b can take part in provoking an autoimmune response. 


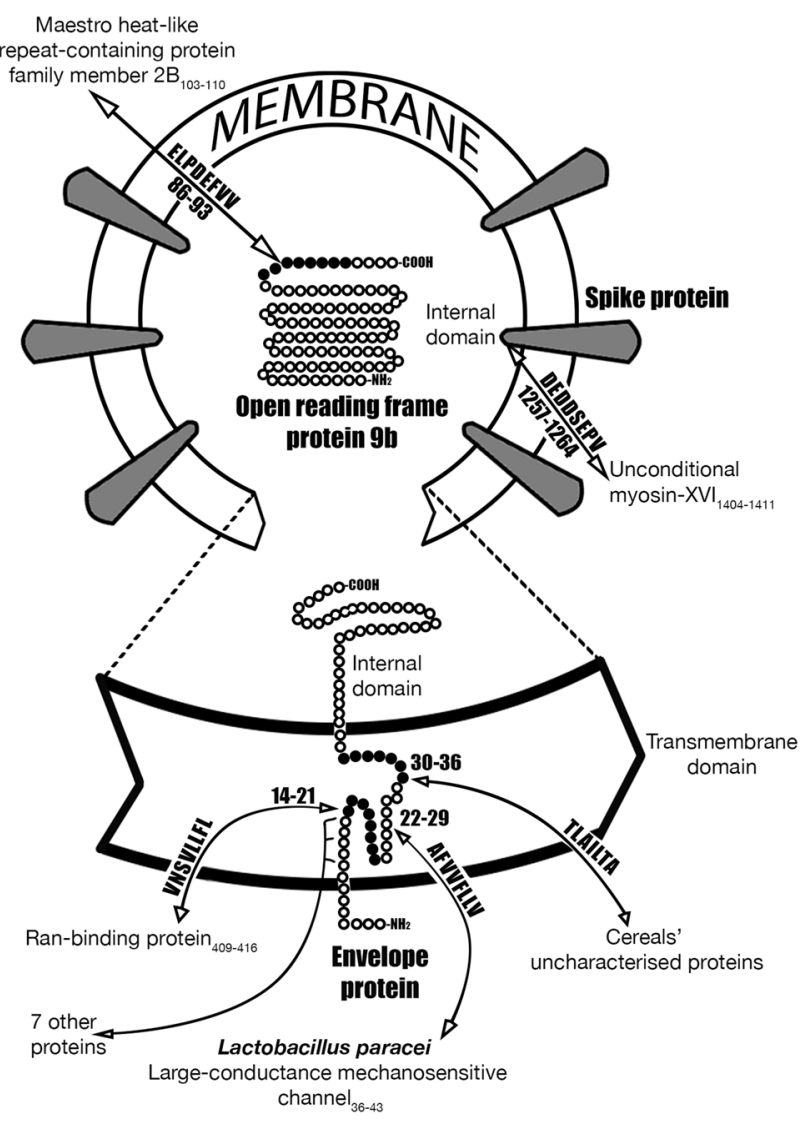

Figure 1. The SARS CoV-2 S, E and ORF9b protein molecules contain hepta/octamers that are homologous to proteins in the human body, including some nutrients and intestinal commensal bacteria.

\begin{tabular}{|c|c|}
\hline In Replicase polyprotein 1a & In human proteins \\
\hline EVEKGVLP $_{55-62}$ & Bifunctional heparan sulfate $\mathrm{N}$-deacetylase/N-sulfotransferase $1_{214-221}$ \\
\hline ESGLKTI L $390-397$ & Annexin $\mathrm{A}_{404-411}$ \\
\hline REETGLLM $_{724-731}$ & Estrogen-related receptor gamma ${ }_{30-37}$ \\
\hline GGSCVLSG $_{1100-1107}$ & Sorting nexin-27 $112-119$ \\
\hline DIQLLKSA $_{1127-1134}$ & Echinoderm microtubule-associated protein-like $1_{38-45}$ \\
\hline RRSFYVYA $_{2431-2438}$ & Transmembrane protein adipocyte-associated $1_{225-232}$ \\
\hline AKKNNLPF $2733-2740$ & Acyl-CoA:lysophosphatidylglycerol acyltransferase $1_{199-206}$ \\
\hline YNYEPLTQ $Q_{3500-3507}$ & DNA helicase $_{199-206}$ \\
\hline SLKELLQN $3530-3537$ & Centromere protein $\mathrm{I}_{496-503}$ \\
\hline DTSLSGFK ${ }_{3671-3678}$ & Solute carrier family 12 member $7_{995-1002}$ \\
\hline PEANMDQE $_{4312-4319}$ & Arachidonate 5-lipoxygenase-activating protein ${ }_{54-61}$ \\
\hline
\end{tabular}

Table 11. Localization of homologous 8-mers in RPP 1a and human proteins. 
MESLVPGFNEKTHVQLSLPVLQVRDVLVRGFGDSVEEVLSEARQHLKDGTCGLVEVEKGVLPQLEQPYVFIKRSDARTAPHGHVMVELVAELEGIQYGRSGETLGVLVPH VGEIPVAYRKVLLRKNGNKGAGGHSYGADLKSFDLGDELGTDPYEDFQENWNTKHSSGVTRELMRELNGGAYTRYVDNNFCGPDGYPLECIKDLLARAGKASCTLSEQLD FIDTKRGVYCCREHEHEIAWYTERSEKSYELQTPFEIKLAKKFDTFNGECPNFVFPLNSI IKTIQPRVEKKKLDGFMGRIRSVYPVASPNECNQMCLSTLMKCDHCGETS WQTGDFVKATCEFCGTENLTKEGATTCGYLPQNAVVKIYCPACHNSEVGPEHSLAEYHNESGLKTILRKGGRTIAFGGCVFSYVGCHNKCAYWVPRASANIGCNHTGVVG EGSEGLNDNLLEILQKEKVNINIVGDFKLNEEIAI ILASFSASTSAFVETVKGLDYKAFKQIVESCGNFKVTKGKAKKGAWNIGEQKSILSPLYAFASEAARVVRS IFSR TLETAQNSVRVLQKAAITILDGISQYSLRLIDAMMFTSDLATNNLVVMAYITGGVVQLTSQWLTNIFGTVYEKLKPVLDWLEEKFKEGVEFLRDGWEIVKFISTCACEIV GGQIVTCAKEIKESVQTFFKLVNKFLALCADS I I IGGAKLKALNLGETEVTHSKGLYRKCVKSREETGLIMPLKAPKEIIFLEGETLPTEVLTEEVVLKTGDLQPLEQPT SEAVEAPLVGTPVCINGLMLLEIKDTEKYCALAPNMMVTNNTFTLKGGAPTKVTFGDDTVIEVQGYKSVNITFELDERIDKVLNEKCSAYTVELGTEVNEFACVVADAVI KTLQPVSELLTPLGIDLDEWSMATYYLFDESGEFKLASHMYCSFYPPDEDEEEGDCEEEEFEPSTQYEYGTEDDYQGKPLEFGATSAALQPEEEQEEDWLDDDSQQTVGQ QDGSEDNQTTTIQTIVEVQPQLEMELTPVVQTIEVNSFSGYLKLTDNVYIKNADIVEEAKKVKPTVVVNAANVYLKHGGGVAGALNKATNNAMQVESDDYIATNGPLKVG GSCVLSGHNLAKHCLHVVGPNVNKGEDIQLLKSAYENFNQHEVLLAPLLSAGIFGADPIHSLRVCVDTVRTNVYLAVFDKNLYDKLVSSFLEMKSEKQVEQKIAEIPKEE VKPFITESKPSVEQRKQDDKKIKACVEEVTTTLEETKFLTENLLLYIDINGNLHPDSATLVSDIDITFLKKDAPYIVGDVVQEGVLTAVVIPTKKAGGTTEMLAKALRKV PTDNYITTYPGQGLNGYTVEEAKTVLKKCKSAFYILPSIISNEKQEILGTVSWNLREMLAHAEETRKLMPVCVETKAIVSTIQRKYKGIKIQEGVVDYGARFYFYTSKTT VASLINTLNDLNETLVTMPLGYVTHGLNLEEAARYMRSLKVPATVSVSSPDAVTAYNGYLTSSSKTPEEHFIETISLAGSYKDWSYSGQSTQLGIEFLKRGDKSVYYTSN PTTFHLDGEVITFDNLKTLLSLREVRTIKVFTTVDNINLHTQVVDMSMTYGQQFGPTYLDGADVTKIKPHNSHEGKTFYVLPNDDTLRVEAFEYYHTTDPSFLGRYMSAL NHTKKWKYPQVNGLTS IKWADNNCYLATALLTLQQIELKFNPPALQDAYYRARAGEAANFCALILAYCNKTVGELGDVRETMSYLFQHANLDSCKRVLNVVCKTCGQQQT TLKGVEAVMYMGTLSYEQFKKGVQIPCTCGKQATKYLVQQESP FVMMSAPPAQYELKHGTFTCASEYTGNYQCGHYKHITSKETLYCIDGALLTKSSEYKGPITDVFYKE NSYTTTIKPVTYKLDGVVCTEIDPKLDNYYKKDNSYFTEQPIDLVPNQPYPNASFDNFKFVCDNIKFADDLNQLTGYKKPASRELKVTFFPDLNGDVVAIDYKHYTPSFK KGAKLLHKPIVWHVNNATNKATYKPNTWCIRCLWSTKPVETSNSFDVLKSEDAQGMDNLACEDLKPVSEEVVENPTIQKDVLECNVKTTEVVGDIILKPANNSLKITEEV GHTDLMAAYVDNSSLTIKKPNELSRVLGLKTLATHGLAAVNSVPWDTIANYAKPFLNKVVSTTTNIVTRCLNRVCTNYMPYFFTLLLQLCTFTRSTNSRIKASMPTTIAK NTVKSVGKFCLEASFNYLKSPNFSKLINIIIWFLLLSVCLGSLIYSTAALGVLMSNLGMPSYCTGYREGYLNSTNVTIATYCTGSIPCSVCLSGLDSLDTYPSLETIQIT ISSFKWDLTAFGLVAEWFLAYILFTRFFYVLGLAAIMQLFFSYFAVHFISNSWLMWLI INLVQMAPISAMVRMYIFFASFYYVWKSYVHVVDGCNSSTCMMCYKRNRATR VECTTIVNGVRRSFYVYANGGKGFCKLHNWNCVNCDTFCAGSTFISDEVARDLSLQFKRPINPTDQSSYIVDSVTVKNGSIHLYFDKAGQKTYERHSLSHFVNLDNLRAN NTKGSLPINVIVFDGKSKCEESSAKSASVYYSQLMCQP ILLLDQALVSDVGDSAEVAVKMFDAYVNTFSSTFNVPMEKLKTLVATAEAELAKNVSLDNVLSTFISAARQG FVDSDVETKDVVECLKLSHQSDIEVTGDSCNNYMLTYNKVENMTPRDLGACIDCSARHINAQVAKSHNIALIWNVKDFMSLSEQLRKQIRSAAKKNNLPFKLTCATTRQV VNVVTTKIALKGGKIVNNWLKQLIKVTLVFLFVAAIFYLITPVHVMSKHTDFSSEIIGYKAIDGGVTRDIASTDTCFANKHADFDTWFSQRGGSYTNDKACPLIAAVITR EVGFVVPGPGTILRTTNGDFLHFLPRVFSAVGNICYTPSKLIEYTDFATSACVLAAECTIFKDASGKPVPYCYDTNVLEGSVAYESLRPDTRYVLMDGSIIQFPNTYLE GSVRVVTTFDSEYCRHGTCERSEAGVCVSTSGRWVLNNDYYRSLPGVFCGVDAVNLLTNMFTPLIQPIGALDISASIVAGGIVAIVVTCLAYYFMRFRRAFGEYSHVVAF NTLLFLMSFTVLCLTPVYSFLPGVYSVIYLYLTFYLTNDVSFLAHIOWMVMFTPLVPFWITIAYIICISTKHFYWFFSNYLKRRVVFNGVSFSTFEEAALCTFLLNKEMY LKLRSDVLLPLTQYNRYLALYNKYKYFSGAMDTTSYREAACCHLAKALNDFSNSGSDVLYQPPQTSITSAVLQSGFRKMAFPSGKVEGCMVQVTCGTTTLNGLWLDDVVY CPRHVICTSEDMLNPNYEDLLIRKSNHNFLVQAGNVQLRVIGHSMQNCVLKLKVDTANPKTPKYKFVRIQPGQTFSVLACYNGSPSGVYQCAMRPNETIKGSFLNGSCGS VGFNIDYDCVSFCYMHHMELPTGVHAGTDLEGNFYGPFVDRQTAQAAGTDTTITVNVLAWLYAAVINGDRWFLNRFTTTLNDFNLVAMKYNYEPLTQDHVDILGPLSAQT GIAVLDMCASLKELLQNGMNGRTILGSALLEDEFTPFDVVRQCSGVTFQSAVKRTIKGTHHWLLLTILTSLLVLVQSTQWSLFFFLYENAFLPFAMGI IAMSAFAMMFVK HKHAFLCLFLLPSLATVAYFNMVYMPASWVMRIMTWLDMVDTSLSGFKLKDCVMYASAVVLLILMTARTVYDDGARRVWTLMNVLTLVYKVYYGNALDQAISMWALI ISV TSNYSGVVTTVMFLARGIVFMCVEYCPIFFITGNTLQCIMLVYCFLGYFCTCYFGLFCLLNRYFRLTLGVYDYLVSTQEFRYMNSQGLLPPKNS IDAFKLNIKLLGVGGK PCIKVATVQSKMSDVKCTSVVLLSVLQQLRVESSSKLWAQCVQLHNDILLAKDTTEAFEKMVSLLSVLLSMQGAVDINKLCEEMLDNRATLQAIASEFSSLPSYAAFATA QEAYEQAVANGDSEVVLKKLKKSLNVAKSEFDRDAAMQRKLEKMADQAMTQMYKQARSEDKRAKVTSAMQTMLFTMLRKLDNDALNNI INNARDGCVPLNI IPLTTAAKL MVVIPDYNTYKNTCDGTTFTYASALWEIOOVVDADSKIVQLSEISMDNSPNLAWPLIVTALRANSAVKLONNELSPVALROMSCAAGTTOTACTDDNALAYYNTTKGGRF VLALLSDLQDLKWARFPKSDGTGTIYTELEPPCRFVTDTPKGPKVKYLYFIKGLNNLNRGMVLGSLAATVRLQAGNATEVPANSTVLSFCAFAVDAAKAYKDYLASGGQP ITNCVKMLCTHTGTGQAITVTPEANMDQESFGGASCCLYCRCH IDHPNPKGFCDLKGKYVQIPTTCANDPVGFTLKNTVCTVCGMWKGYGCSCDQLREPMLQSADAQSFL NGFAV

Replicase polyprotein RPP 1a. Replicase polyprotein RPP 1a, 4405 aa.

The longest $n$-mers are underlined.

In the RPP 1a molecule, we localized eleven 8-mers (Table 11) and more than a hundred 7-mers homologous to human proteins.

Some of the 8-mers are found in more than one human protein, some fold into long $\mathrm{n}$-mers, for example EDIQLLKSAYENENQH ${ }_{1126-1141}$, EVEKGVLPQLEQPY ${ }_{55-68}$ and SVEEVLSEARQHL ${ }_{34-46}$.

In the RPP 1a molecule, 7-mers SCGNFKV 505-511 and AIFYLIT $2785-2791$ are homologous to human olfactory receptor proteins $52 \mathrm{~N} 2_{190-196}$ and $2 \mathrm{~W} 1_{32-38}$, respectively. A heptamer LKTLLSL $\mathrm{LLF}_{1556-1562}$ is homologous to the human bitter taste receptor T2R55 $5_{181-187}$ (Fig. 2).

Replicase polyprotein RPP 1ab. This huge (7096 aa; the primary structure see in ${ }^{18}$ ) molecule contains 210 heptaand octamers homologous to human proteins. Some of them fold into long (more than $15 \mathrm{aa}$ ) n-mers.

The possibility of the involvement of replicases in provoking an autoimmune response is debatable. Enzymes in general, and cell cycle enzymes in particular, are evolutionarily highly conserved. Fragments homologous to human proteins must be thrown in huge quantities into the gut lumen during the decay of any microorganism that dies there. It is possible that the interaction of replicases with the host's immune system obeys the laws other than for shorter proteins.

ORF6, ORF10, and ORF14. In these polypeptides (61, 38, and 73 aa, respectively), we did not find 7-/8-mers homologous to human proteins. When assessing the role of SARS CoV-2 proteins in mimicry and provoking an autoimmune response in humans, we considered the following parameters: (i) the number of homologous n-mers; (ii) the compactness of their arrangement in the SARS CoV-2 protein molecules; (iii) intradomain localization (external, transmembrane, internal) of the SARS CoV-2 proteins, and (iv) physiological functions that involve the homologous human proteins (Table 12).

\section{Conclusions}

Analysis of homology between the SARS CoV-2 and human proteins led us to the following conclusions. Some of the SARS CoV-2 proteins can be implicated in mimicry that can delay the response of innate immunity to the invasion of virus particles into a macroorganism, and in provoking an autoimmune process that directs a part 


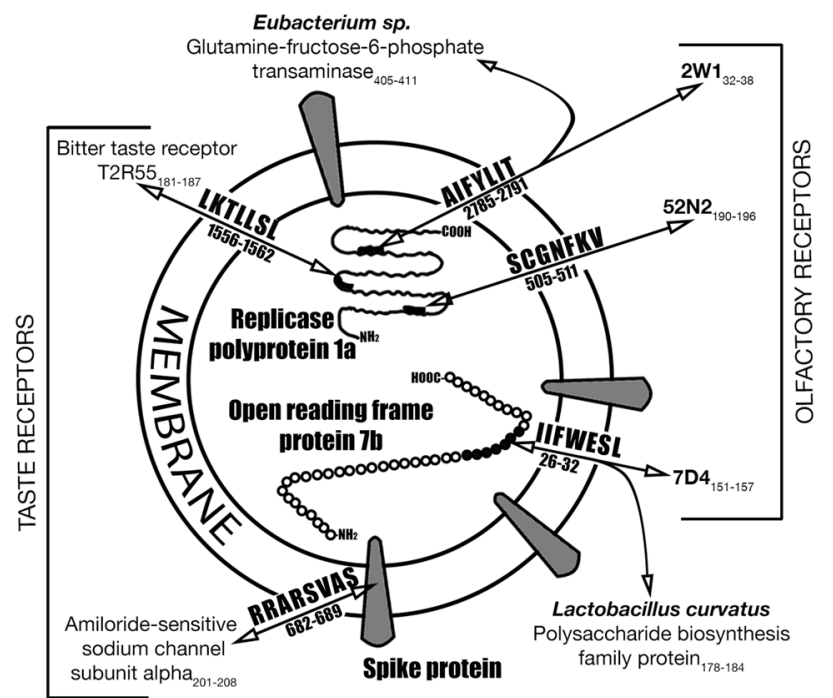

Figure 2. Some SARS CoV-2 hepta/octamers are homologous to human olfactory and taste receptor proteins. Homology to some proteins of commensal gut bacteria is also shown.

\begin{tabular}{|c|c|c|c|c|}
\hline Goup of proteins & Protein & Mimicry & Autoimmune response & Comment \\
\hline \multirow{4}{*}{ Structural } & S & +++ & + & $\begin{array}{l}\text { Taste?-Amiloride-sensitive sodium channel subunit alpha }{ }_{201-208} \\
\text { Muscle contraction?-Unconventional myosin-XVI }{ }_{1404-1421}\end{array}$ \\
\hline & E & - & +++ & $\begin{array}{l}\text { Gut microbiota?_Lactobacillus paracasei } \\
\text { Digestion?-Cereals' proteins }\end{array}$ \\
\hline & M & ++ & - & \\
\hline & $\mathrm{N}$ & - & ++ & \\
\hline \multirow{10}{*}{ Nonstructural } & ORF3a & - & + & \\
\hline & ORF6 & - & - & No homology \\
\hline & ORF7a & - & + & \\
\hline & ORF7b & - & + & $\begin{array}{l}\text { Smell?-Olfactory receptor 7D4 } \\
\text { Gut microbiota?-Lactobacillus curvatus }\end{array}$ \\
\hline & ORF8 & - & ++ & \\
\hline & ORF9b & - & ++ & $\begin{array}{l}\text { Sperm capacitation?--Maestro heat-like repeat-containing } \\
\text { protein family member } 2 \mathrm{~B}_{103-110}\end{array}$ \\
\hline & ORF10 & - & - & No homology \\
\hline & ORF14 & - & - & No homology \\
\hline & RPP1a & - & $?$ & $\begin{array}{l}\text { Taste?-T2R55 receptor } \\
\text { Smell?-OOlfactory receptors } 2 \mathrm{~W} 1 \text { and } 52 \mathrm{~N} 2 \\
\text { Gut microbiota?-Eubacterium sp. }\end{array}$ \\
\hline & RPPlab & - & $?$ & \\
\hline
\end{tabular}

Table 12. Qualitative assessment of the possibility for the SARS CoV-2 proteins to be involved in the processes of mimicry and provoking an autoimmune response.

of the immune response to the proteins of a macroorganism (after the destruction of virus particles). Mimicry is probably more characteristic of the spike (S) protein, and the provocation of an autoimmune response seems to be a distinctive feature of the envelope (E) protein. The ORF7b protein may be involved in the impairment of olfactory receptors, and the $\mathrm{S}$ protein may be involved in taste perception dysfunction.

Drugs aimed at destructing or blocking these and alike regions in proteins of SARS CoV-2 and other viruses can enable the human immune system not to succumb to viral deception and destroy the invader shortly after its penetration into a macroorganism. It should also be borne in mind that drugs affecting such imitation regions can damage native proteins present of the human body. Destroying or blocking such regions can weaken the autoimmune response. 


\section{Data availability}

The highest.

\section{Code availability}

Source code of Ouroboros (v. 0.5) is fully available at github. URL: https://github.com/liquidbrainisstrain/ourob oros. Artwork: We used GIMP (Version 2.10.22) to create our artwork. The figures are completely original and have not been published anywhere.

Received: 12 April 2021; Accepted: 2 August 2021

Published online: 25 August 2021

\section{References}

1. Sanami, S. et al. Design of a multi-epitope vaccine against SARS-CoV-2 using immunoinformatics approach. Int. J. Biol. Macromol. 164, 871-883. https://doi.org/10.1016/j.ijbiomac.2020.07.117 (2020).

2. Lederberg, J. Infectious history. Science 288(5464), 287-293 (2000).

3. Terekhov, A. Ouroboros (Version 0.5) [Source code]. https://github.com/liquidbrainisstrain/ouroboros.

4. Proteomes: Severe acute respiratory syndrome coronavirus 2 (2019-nCoV) (SARS-CoV-2). https://www.uniprot.org/proteomes/ UP000464024 SARS-COV-2, accessed 20 Aug 2020.

5. Proteomes: Homo sapiens (Human). https://www.uniprot.org/proteomes/UP000005640 Homo sapiens, accessed 03 Sept 2020.

6. Koralnik, I. J. \& Tyler, K. L. COVID-19: A global threat to the nervous system. Ann. Neurol. 88(1), 1-11. https://doi.org/10.1002/ ana.25807 (2020).

7. Huang, T. \& Stähler, F. Effects of dietary $\mathrm{Na}^{+}$deprivation on epithelial $\mathrm{Na}^{+}$channel (ENaC), BDNF, and TrkB mRNA expression in the rat tongue. BMC Neurosci. 10, 19. https://doi.org/10.1186/1471-2202-10-19 (2009).

8. Mandala, V. S. et al. Structure and drug binding of the SARS-CoV-2 envelope protein transmembrane domain in lipid bilayers. Nat. Struct. Mol. Biol. 27(12), 1202-1208. https://doi.org/10.1038/s41594-020-00536-8 (2020).

9. Li, S. W. et al. Gene editing in CHO cells to prevent proteolysis and enhance glycosylation: Production of HIV envelope proteins as vaccine immunogens. PLoS ONE 15, e0233866. https://doi.org/10.1371/journal.pone.0233866 (2020).

10. Rathore, A. S., Sarker, A. \& Gupta, R. D. Production and immunogenicity of Fubc subunit protein redesigned from DENV envelope protein. Appl. Microbiol. Biotechnol. 104, 4333. https://doi.org/10.1007/s00253-020-10541-y (2020).

11. Ho, J. K., Jeevan-Raj, B. \& Netter, H. J. Hepatitis B Virus (HBV) subviral particles as protective vaccines and vaccine platforms. Viruses 12, 126. https://doi.org/10.3390/v12020126 (2020).

12. Abdelmageed, M. I. et al. Design of a multiepitope-based peptide vaccine against the E protein of human COVID-19: An immunoinformatics approach. Biomed. Res. Int. 2020, 2653286. https://doi.org/10.1155/2020/2683286 (2020).

13. DeDiego, M. L. et al. Inhibition of NF- $\kappa B$-mediated inflammation in severe acute respiratory syndrome coronavirus-infected mice increases survival. J. Virol. 88, 913-924 (2014).

14. Regla-Nava, J. A. et al. Severe acute respiratory syndrome coronaviruses with mutations in the E protein are attenuated and promising vaccine candidates. J. Virol. 89, 3870-3887 (2015).

15. Hassan, S. S. et al. A unique view of SARS-CoV-2 through the lens of ORF8 protein. Comput. Biol. Med. 133, 104380. https://doi. org/10.1016/j.compbiomed.2021.104380 (2021).

16. MROH2B: Function. https://www.nextprot.org/entry/NX_Q7Z745.

17. Sansone, A. et al. Addressing male sexual and reproductive health in the wake of COVID-19 outbreak. J. Endocrinol. Invest. 44(2), 223-231. https://doi.org/10.1007/s40618-020-01350-1 (2021).

18. Replicase polyprotein $1 \mathrm{ab}$ [Severe acute respiratory syndrome coronavirus 2]. https://www.ncbi.nlm.nih.gov/protein/P0DTD1.1? report=fasta.

\section{Author contributions}

A.M. and V.K. wrote the main manuscript text. A.T. and D.K. prepared data analysis. All authors reviewed the manuscript.

\section{Funding}

This research is an authors' initiative project funded exclusively from their personal sources.

\section{Competing interests}

The authors declare no competing interests.

\section{Additional information}

Correspondence and requests for materials should be addressed to A.M.

Reprints and permissions information is available at www.nature.com/reprints.

Publisher's note Springer Nature remains neutral with regard to jurisdictional claims in published maps and institutional affiliations.

(c) (i) Open Access This article is licensed under a Creative Commons Attribution 4.0 International License, which permits use, sharing, adaptation, distribution and reproduction in any medium or format, as long as you give appropriate credit to the original author(s) and the source, provide a link to the Creative Commons licence, and indicate if changes were made. The images or other third party material in this article are included in the article's Creative Commons licence, unless indicated otherwise in a credit line to the material. If material is not included in the article's Creative Commons licence and your intended use is not permitted by statutory regulation or exceeds the permitted use, you will need to obtain permission directly from the copyright holder. To view a copy of this licence, visit http://creativecommons.org/licenses/by/4.0/.

(C) The Author(s) 2021 